\title{
Centros de Apoyo y Desarrollo Educativo Profesional para la observación y disminución de la deserción universitaria
}

\author{
William Oswaldo Flores López ${ }^{1}$, Emmanuelle Gutiérrez y Restrepo ${ }^{2}$, Olga Lucía León Corredor ${ }^{3}$, \\ Joao Sarraipa ${ }^{4}$, Celson Pantoja Lima ${ }^{5}$, Cristian Merino ${ }^{6}$, Dora Inés Calderón 7 , Marisa Guinocchio ${ }^{8}$, \\ Mailing Rivera Lam ${ }^{9}$, María Eugenia Calderón ${ }^{10}$ y Jesús G. Boticario ${ }^{11}$
}

\section{Resumen}

Este artículo ha presentado el estado del arte de la deserción universitaria en Europa y América Latina, y el modelo de Centro de Apoyo y Desarrollo Educativo Profesional (CADEP) para observar y tratar de disminuir la deserción estudiantil. Fue un estudio cualitativo con un enfoque hermenéutico donde se analizaron textos de libros, artículos científicos, informes de congresos, artículos de prensa, leyes, políticas y normas institucionales. Los resultados mostraron: que la deserción universitaria en Europa, América Latina y el Caribe oscila del $30 \%$ al 60\%, centrada en factores como lo económico, familiar, ambiente pedagógico-didáctico, motivacional y afectivos, y que todos estos factores influyen en el rendimiento académico del estudiantado y propician el abandono de la universidad. Todo este planteamiento, permite al proyecto ACACIA ofrecer un sistema articulado de atención integral, que parte de un enfoque diferencial y con acciones que cubre no sólo las necesidades del estudiantado (de accesibilidad y afectividad en la experiencia de enseñanza y aprendizaje) y sus preferencias, sino también acciones y formación dirigidas al profesorado como al personal técnico y administrativo universitario, sin olvidar a la familia y el entorno social en el que se inscribe la universidad incluyendo al tejido empresarial, con una perspectiva integradora y aplicada de la innovación desarrollada.

1 Doctor en Educación, Profesor, Investigador y Gestor de Proyectos de la Universidad de las Regiones Autónomas de la Costa Caribe Nicaragüense, Nicaragua. Email: william.flores@uraccan.edu.ni

2 Doctora en Ciencias de la Comunicación. Profesora e Investigadora del grupo aDeNud en la Universidad Nacional a Distancia, España. Email: emmanuelle@sidar.org

3 Doctora en Educación, Profesora e Investigadora de la Universidad Distrital Francisco José de Caldas, Colombia. Email: oleoncorredor@gmail.com

4 Doctor en Sistemas Industrial de Información, Investigador del Centro de Tecnología y Sistema la Universidad Nova de Lisboa, Portugal. Email: jfss@uninova.pt

5 Doctor en Electrónica, Investigador de la Universidade Federal do Oeste do Pará, Brasil. Email: celson.ufopa@gmail.com

6 Doctor en Didáctica de las Ciencias Experimentales, Profesor e Investigador de la Pontifica Universidad Católica de Valparaíso, Chile. Email: cristian.merino@ucv.cl

7 Doctora en Educación, Profesora e Investigadora de la Universidad Distrital Francisco José de Caldas. Email: dorainescalderon@gmail.com

8 Magister en Lingüística, Profesora e Investigadora de la Universidad Nacional Mayor de San Marcos, Perú. Email: marisaginocchio1@gmail.com

9 Doctora en Antropología, Profesora e Investigadora de la Universidad de Antofagasta, Chile. Email: mailing.rivera@uantof.cl

10 Doctora en Ingeniería Química, Profesora e Investigadora de la Universidad Distrital Francisco José de Caldas, Colombia. Email: mcalderon@udistrital.edu.co

11 Doctor en Física, Vicerrector en Innovación y Desarrollo Tecnológico de la Universidad Nacional a Distancia, España. Email: jgb@dia.uned.es 
Palabras clave: Deserción universitaria; accesibilidad; afectividad; retención universitaria.

\section{Summary}

This article presents the state of art of the college desertion in Europe and Latin America, as well as the model of the Support Center and Professional Educational Development (CADEP) in order to observe and try to reduce student's desertion. The study was qualitative with a hermeneutic approach in which we analyzed different texts books, scientific articles, congress reports, press articles, laws, policies and institutional rules. The results revealed that the college desertion in Europe, Latin America and the Caribbean ranges from $30 \%$ to $60 \%$, and it is focus on factors such as economic, family, educational, motivational and emotional environment, but most importantly is that all these factors influence the academic performance of students and contribute to the desertion from the university. This whole approach allows the ACACIA project to provide an articulated system of integral attention, that is based on differential approach which includes actions covering not only the needs of the students (accessibility and affection in the experience of teaching and learning) and its preferences, but also actions and training aimed at teachers as well as to the technical and administrative university staff, not forgetting family and the social environment in which the university is a fundamental part, including the business sector, with an integrated perspective of the developed innovation.

Keywords: College desertion; accessibility; affectivity; college retention.

\section{Introducción}

Existe una preocupación en la comunidad de investigadores de Europa y América Latina sobre el por qué el estudiantado universitario abandona sus estudios por factores emocionales, económicos, marginación cultural, social o discapacidad, recursos didácticos, brechas de comunicación e intercambio entre el profesorado. Por tales razones, se realiza un análisis sobre la deserción universitaria en Europa, América Latina y el Caribe, así como la estrategia de ACACIA para la implementación de centros de apoyo y desarrollo educativo profesional, asumiendo que el principio de una educación para todos reconoce: el valor de cada persona en el sistema educativo; el desarrollo académico de cada uno de sus integrantes, organiza espacios para acoger a personas en situación de vulnerabilidad para su formación y desarrollo profesional en el ambiente universitario; considera la dimensión afectiva y emocional de cada uno de sus integrantes y fomenta el apoyo institucional a los grupos que requieren un acompañamiento específico para su formación. 


\section{Revisión de literatura}

\section{La deserción estudiantil en las instituciones de Educación Superior}

La deserción se puede definir como el proceso de abandono, voluntario o forzoso de la carrera en la que se matricula un estudiante, por la influencia positiva o negativa de circunstancia internas o externa a él o ella (González, 2005). Frente a las causas asociadas a la deserción, estas están determinadas por factores multivariados, que son agrupados en cuatro categorías globales, estas son: personales, académicas, socioeconómicas e institucionales (Parrino, 2014). En cambio, Tinto (2009) afirma que la deserción va en dependencia de su trayectoria académica, y la clasifica según el período en el que se presente: deserción precoz, deserción temprana y deserción tardía. La primera en el período comprendido entre la inscripción y la admisión, una de las razones que genera este tipo de deserción es la falta de conocimiento sobre el programa académico o la universidad, o el trato percibido por parte de la institución en el proceso; la segunda corresponde a la deserción durante los primeros semestres académicos; $y$ la tercera se refiere al abandono de los estudios por parte del estudiantado durante los semestres finales.

\section{Duración}

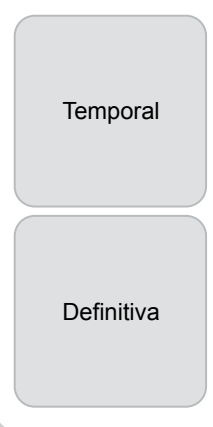

\section{Temporalidad}

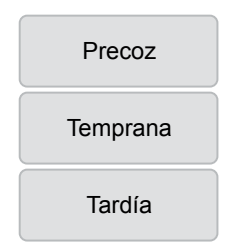

Alcance

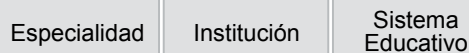

Factores personales

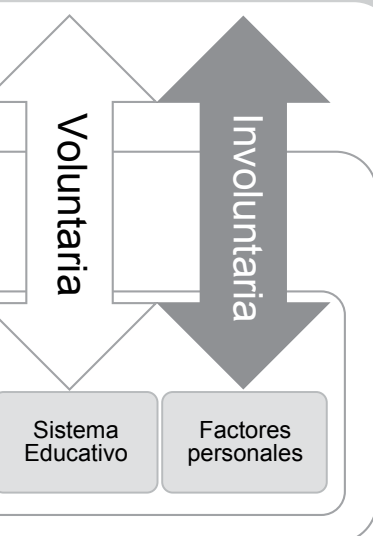

Figura No. 01. Tipología de la deserción universitaria

Por otra parte, también es delimitada según el mecanismo de abandono, cuando el estudiante toma la decisión de abandonar su proceso formativo se conoce como deserción voluntaria; pero cuando es la institución la que no permite al estudiante continuar con la carrera se habla de deserción forzada, este fenómeno responde al alcance de la misma, por ello se puede delimitar en una deserción del programa (cambio de carrera), de la institución (cambio de universidad) y del sistema educativo (desvinculación completa) (Sierra \& Hernández, 2014). Pero la deserción no comprende 
solamente una agrupación de factores de riesgo, sino que también se manifiesta por causas individuales de deserción relacionadas con la baja autoestima, la automotivación, los hábitos de estudio, la persistencia y tolerancia a la frustración, las expectativas y valores personales propios del estudiante, que le dificulta adaptarse a la institución, el proceso de aprendizaje (Casiano, 2004).

Recientemente y centrado en el área latinoamericana, el proyecto europeo Gestión Universitaria Integral del Abandono (GUIA) del programa ALFA-III (Programa Alfa, 2012), que tuvo una duración de tres años (2011'2014) y que continúa trabajando a través de la organización del congreso anual CLABES, ha analizado los ratios y causas de deserción, proponiendo modelos de relaciones causales entre diversas variables de carácter social, económico, académico o psicológico y la decisión de abandonar. Los resultados de los estudios llevados a cabo durante el proyecto les llevan a la conclusión de que las principales causas o factores incidentes en la deserción pueden agruparse en cuatro categorías: las externas al sistema de Educación Superior, las propias del sistema e institucionales, las causas académicas y las de carácter personal.

Entre las causas externas, consideran que las principales son: las condiciones socioeconómicas tanto del estudiante como del grupo familiar (el lugar de residencia; el nivel de ingresos; el nivel educativo de los padres; el ambiente familiar, la necesidad de trabajar para mantenerse o aportar a su familia). Por tanto, la situación económica y la eficiencia en el gasto son cuestiones críticas. Entre las causas propias del sistema e institucionales, destacan: el incremento de la matrícula, particularmente en los quintiles de menores ingresos que requieren de mayor apoyo debido a su deficiente preparación previa; la carencia de mecanismos adecuados de financiamiento del sistema, en especial para el otorgamiento de ayudas estudiantiles, créditos y becas; las políticas de admisión (abierta o selectiva); el desconocimiento de la profesión y de la metodología de las carreras; el ambiente educativo e institucional y la carencia de lazos afectivos con la universidad.

Entre las causas de orden académico, consideran: la formación académica previa, los exámenes de ingreso, el nivel de aprendizaje adquirido, la excesiva orientación teórica y la escasa vinculación de los estudios con el mundo laboral, la falta de apoyo y orientación recibida por los profesores, la falta de información al elegir la carrera, la carencia de preparación para el aprendizaje y reflexión autónoma, la excesiva duración de los estudios, la heterogeneidad del estudiantado y la insuficiente preparación de los profesores para enfrentar la población estudiantil que actualmente ingresa a las universidades. En términos concretos, en uno de los estudios se constataba que las principales diferencias entre desertores y no desertores eran: la preferencia en la elección de la carrera (18\% de diferencia); las calificaciones de la enseñanza secundaria (18\%); y el puntaje en las pruebas de selección (13\%). 
Entre las causas personales, enumeran aspectos de orden motivacional y actitudinal tales como: la actividad económica del estudiante, sus aspiraciones y motivaciones personales, la disonancia con sus expectativas, su insuficiente madurez emocional, las aptitudes propias de su juventud, el grado de satisfacción con la carrera, las expectativas al egreso de la carrera en relación con el mercado laboral, las dificultades personales para la integración y adaptación, la dedicación del alumno, la falta de aptitudes, y las habilidades o interés por la carrera escogida.

\section{Modelos de centros de apoyo a la comunidad universitaria}

El modelo de los centros de apoyo y desarrollo profesional que aquí presentamos, es el resultado del análisis de múltiples aproximaciones teóricas que tratan los problemas de retención estudiantil, Summerskill (1962), Astin (1977, 1993), Bean (1980, 1983), Bean y Metzner (1985) y Spady (1971) entre otros. Estudios realizados por el Centro para la Retención Estudiantil -CSCSR-indican que a pesar de los esfuerzos de las universidades en políticas de retención no se ha logrado aumentar las tasas de retención. Las principales estrategias propuestas por el CSCSR tienen que ver con la creación de un comité de retención en las instituciones, la identificación de estudiantes con riesgo de deserción, la identificación de problemas dentro del aula de clase tales como poca atención, obtención de malas calificaciones y poca participación en clase, entre otros. Los resultados del proyecto GUIA hacen una serie de propuestas para disminuir la deserción universitaria, dicha estrategia se clasifica en tres niveles:

En el nivel de los sistemas de Educación Superior proponen: profundizar los métodos básicos de medición, mejorar los sistemas y pruebas de selección, el diseño de observatorios laborales, los sistemas de información pública, la definición de estándares de calidad y el énfasis en la eficiencia académica en los procesos de evaluación. Plantean también, mejorar la articulación con la Educación Secundaria, facilitar la movilidad institucional, y otorgar a los estudiantes mayor apoyo financiero y becas.

En el nivel institucional y académico sugieren: mejorar los mecanismos de detección temprana de potenciales desertores, identificar grupos de riesgo, otorgar apoyo tutorial integral al estudiante, mejorar la orientación vocacional, realizar seguimiento estudiantil y mejorar la administración curricular otorgando certificaciones tempranas y creando salidas intermedias.

En el nivel pedagógico, plantean: incrementar la autoestima y autoconocimiento, crear redes de apoyo, trabajar la motivación y autodeterminación, perfeccionar los procesos cognitivos y metacognitivos, incorporar el manejo y control de la ansiedad, considerar los estilos de aprendizaje, la atención, la concentración y los distintos tipos de inteligencias. Recomiendan también «incorporar sistemas de nivelación y procesos remediales - por ejemplo, cursos de nivelación para los estudiantes que no aprueban los exámenes de ingreso y complementar las deficiencias de la Educación Secundaria 
en materias fundamentales-, crear ciclos generales de conocimientos básicos, generar condiciones adecuadas para el aprendizaje - métodos, infraestructura y recursos, desarrollar innovaciones curriculares- perfiles y enseñanza por competencias y fortalecer la metodología de resolución de problemas), implementar currículos más flexibles, establecer una titulación directa con un trabajo de investigación desarrollado en el último año, promover cambios metodológicos, incorporar TIC, lograr el perfeccionamiento pedagógico de los docentes y mejorar los procesos de evaluación. En esa línea, han sugerido desarrollar acciones tendentes a: la detección temprana de posibles desertores e identificar los momentos críticos, informar y orientar sobre las distintas ramas científicas con énfasis en aspectos de destrezas requeridas, así como del mercado profesional, acordar convenios con sectores productivos para prácticas laborales y para la realización de investigaciones, establecer un diseño y administración curricular apropiado, generar procesos administrativos eficientes y ofrecer orientación al estudiante con riesgos de desertar.

Como veremos, los CADEP Acacia cubren todas estas estrategias, pero además lo hace de manera articulada y va aún más allá.

\section{Materiales y métodos}

El presente estudio se enmarca en el paradigma cualitativo porque es una actividad sistemática orientada a la comprensión en profundidad de fenómenos educativos y sociales, a la transformación de prácticas y escenarios socioeducativos, a la toma de decisiones y también hacia el descubrimiento y desarrollo de un cuerpo organizado de conocimiento. Desde esta perspectiva, Sierra, H., \& Hernández, O. (2014), explican que en el paradigma cualitativo el enfoque o diseño "es el abordaje general que utilizará el investigador en el proceso de investigación” (p. 492). En este sentido, esta investigación tiene un enfoque hermenéutico, porque se interpretaron los significados de textos y acciones en el proceso educativo.

Por tales razones, se utilizó como método el análisis de contenido de los textos, el cual ayudó a describir los documentos en sus partes esenciales. Para ello, se empleó la técnica de revisión de documentos, donde nos centramos en los argumentos, razones, propósitos y los significados que los mismos presentan sobre la deserción universitaria. Por consiguiente, se construyó una guía de revisión documental como instrumento, que permitió analizar los libros, artículos científicos, artículos de prensa, leyes, políticas y normas institucionales. 


\section{Resultados y discusión}

\section{Niveles de deserción universitaria en Europa, América Latina y el Caribe}

Los niveles de abandono universitario en España que venían oscilando entre el $30 \%$ y el $50 \%$ se han reducido en el 2015 a $21.9 \%$, lo que aún sigue siendo la cifra más alta de toda la Unión Europea. Tras España, los países de la Unión Europea con ratios más altos de abandono sin completar los estudios universitarios de jóvenes entre 18 y 24 años son Malta (20.4\%), Rumanía (18.1\%) y Portugal (17.4\%); teniendo el porcentaje más bajo Croacia (4.3\%). El objetivo de la Unión Europea para el 2020 es conseguir un ratio de deserción del $10 \%$ y aumentar la proporción de la población de 30 a 34 años que finalice la enseñanza superior o equivalente a por lo menos el 40\%. En este contexto el objetivo español es reducir la deserción hasta al $15 \%$ (Eurostat, 2015).

En el caso de América Latina las cifras son similares. Por ejemplo, en Colombia la deserción tiene un nivel de abandono cercano al 50\% según el Ministerio de Educación Nacional de Colombia, está problemática de la deserción en la Educación Superior colombiana, hoy enunciada como promoción de la permanencia y la graduación, ha sido temática de política pública acogida por las instituciones de Educación Superior, a través de la cual hoy se cuenta no sólo con información actualizada y contextualizada de los estudiantes de pregrado del país, sino con una perspectiva pedagógica direccionada por el paradigma de la educación inclusiva.

En Argentina ha llegado al 60\%, según la Secretaria de Políticas Universitarias del Ministerio de Educación de la Nación; hay

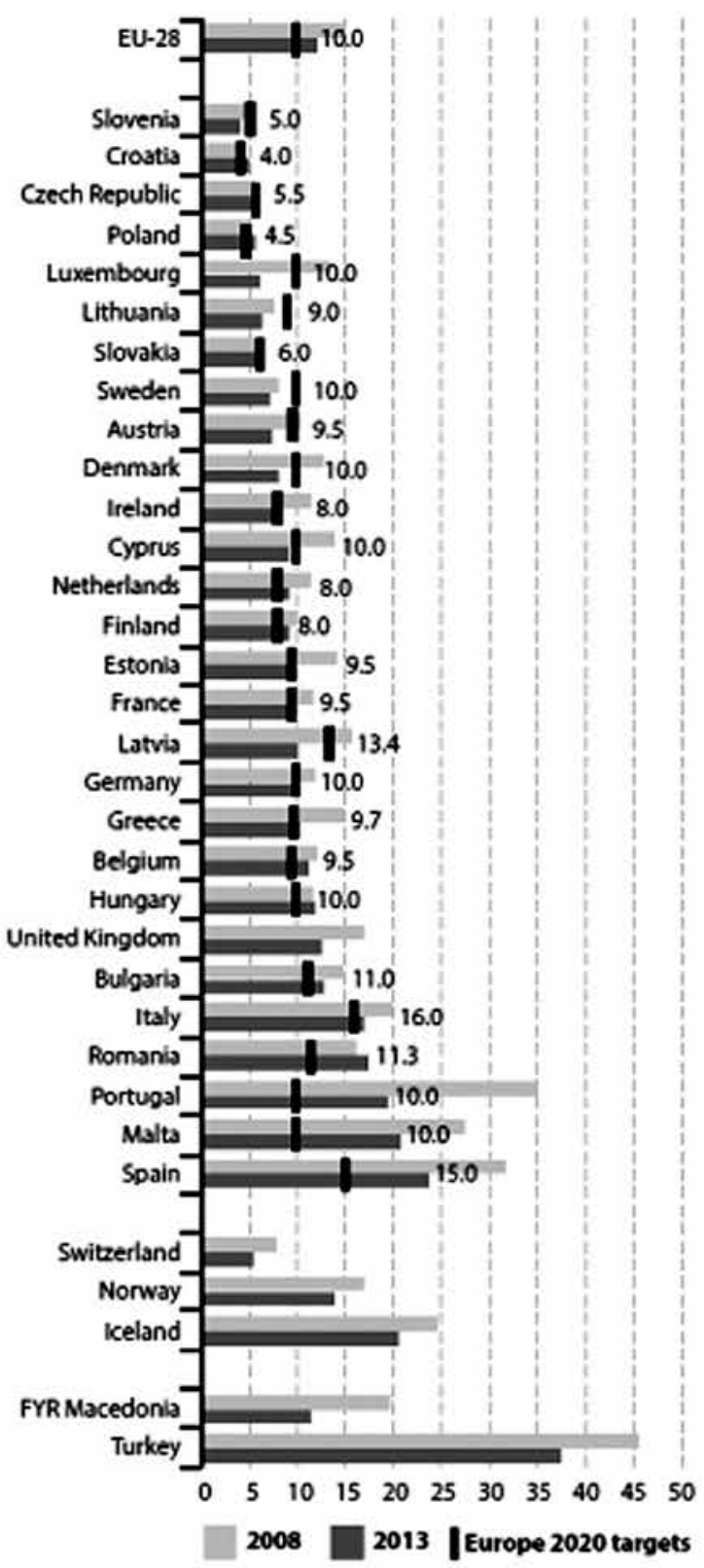

(4) Bresk in series for LU (2009), NLL 12010), LV (2011) and FR (2013)

National targets: EU-28, DK, DE, LU, SE: under 10\%, ES: $15 \%$ idrop-out ratel, NL: under $8 \%$, LT: Under $9 \%$, SK: under $6 \%$, UK-no target.

Figura No. 02. Niveles de abandono en Europa y objetivo para el 2020 
casos con niveles mucho más bajos como por ejemplo Nicaragua, donde apenas llega al $30 \%$, dicha deserción, obedece a indicadores relacionados con aspectos como: académicos, económicos, motivacionales, normativos, ambiente familiar, orientación profesional y proceso de acompañamientos al estudiante en su formación (Aburto, 2013). Estos indicadores han sido trabajados por las Instituciones de Educación Superior de Nicaragua a través de acciones institucionales (ambientes académicos y familiares, becas, orientación profesional y conversatorios con el estudiantado), pero sigue siendo insuficientes los esfuerzos para lograr que todas las personas que ingresan al sistema de Educación Superior concluyan exitosamente sus estudios.

En Chile, más del 50\% de quienes se matriculan en la Educación Superior no concluyen el programa en el que se matricularon inicialmente. Esta situación genera importantes pérdidas de eficiencia para el Estado y las instituciones, así como disminución de oportunidades para los estudiantes y sus familias (MINEDUC, 2012). Por otro lado, el problema del abandono ha afectado tradicionalmente a los estudiantes de universidad (Cabrera, Bethencourt, Álvarez Pérez y González Afonso, 2006), especialmente en la enseñanza a distancia (Moncada, 2014) y últimamente se ha incrementado en el creciente uso de los cursos masivos abiertos (Lukeš, 2012).

La Agenda Educativa post-2015 de la UNESCO evidencia problemáticas que afectan a la Educación Superior en América Latina y del Caribe (ALC) de las que se derivan las siguientes situaciones críticas (UNESCO, 2014): deserción estudiantil por factores emocionales, académicos, de marginación cultural, económica o social, o por discapacidad; falta de recursos didácticos en profesores universitarios para responder a exigencias de estudiantes en situación de vulnerabilidad; existencia de brechas de comunicación y cooperación entre profesores, investigadores, administrativos y directivos que impiden gestar acciones colectivas para enfrentar problemas transversales vinculados al acceso y la permanencia exitosa en la universidad.

Todos estos factores, sumados a otros de índole personal y afectivo, influyen considerablemente en el nivel académico de los alumnos universitarios. En este sentido, ACACIA contará con tres centros pilotos en Perú, Colombia y Nicaragua, que constituyen un dispositivo tipo red que permitirá a:

- La región ALC y a cada uno de estos países tener un observatorio de deserción estudiantil que proporcione estadísticas y relaciones entre estados afectivos de los estudiantes, estrategias didácticas-tecnológicas y disminución de la deserción. Es decir, se contará con una herramienta precisa para enfrentar el fenómeno de la deserción.

- Las universidades de la región tener un modelo en ejecución para la creación de centros similares con sistemas de laboratorios, que fortalecen la formación y la creación de soluciones innovadores en tecnología educativa, en didácticas accesibles, en usos de sistemas de detección de emociones y de recursos innovadores. Es 


\section{EDUCACIÓN SUPERIOR}

decir, existe un espacio propicio para la interacción de grupos interinstitucionales que aúnan esfuerzos en soluciones a problemas educativos comunes.

- Los profesores, estudiantes investigadores, administrativos y directivos de estas tres universidades y de las universidades socias, disponer de un espacio institucional, que ofrece recursos para su actualización profesional en procesos de aprendizaje de poblaciones vulnerables y el desarrollo de un perfil profesional innovador y único en la región. Es decir, son profesionales empoderados en la educación accesible, afectiva e inclusiva.

- La existencia de un diseño de «CADEP Acacia» con los correspondientes diseños de cada módulo que entrega el proyecto, permite a las universidades de la región tener un modelo replicable que además de ofrecer una estructura confiable, acelera los procesos institucionales de creación de un CADEP Acacia.

\section{Los CADEP}

Los Centros de Apoyo y Desarrollo Educativo Profesional (CADEP), son organismos que apoyan, cultivan, adaptan, comunican, innovan, acogen (ACACIA) las experiencias, recursos, equipos, problemas $y$, soluciones que requieren las IES para el fomento profesional de todos sus miembros, para la disminución de la deserción estudiantil, para la modernización de sus estructuras organizativas y para el respeto por el otro y sus diferencias. Esta estructura es una nueva herramienta institucional para detectar, estudiar y solucionar problemas que una dependencia, una facultad un programa de formación profesional no pueden enfrentar de manera aislada.

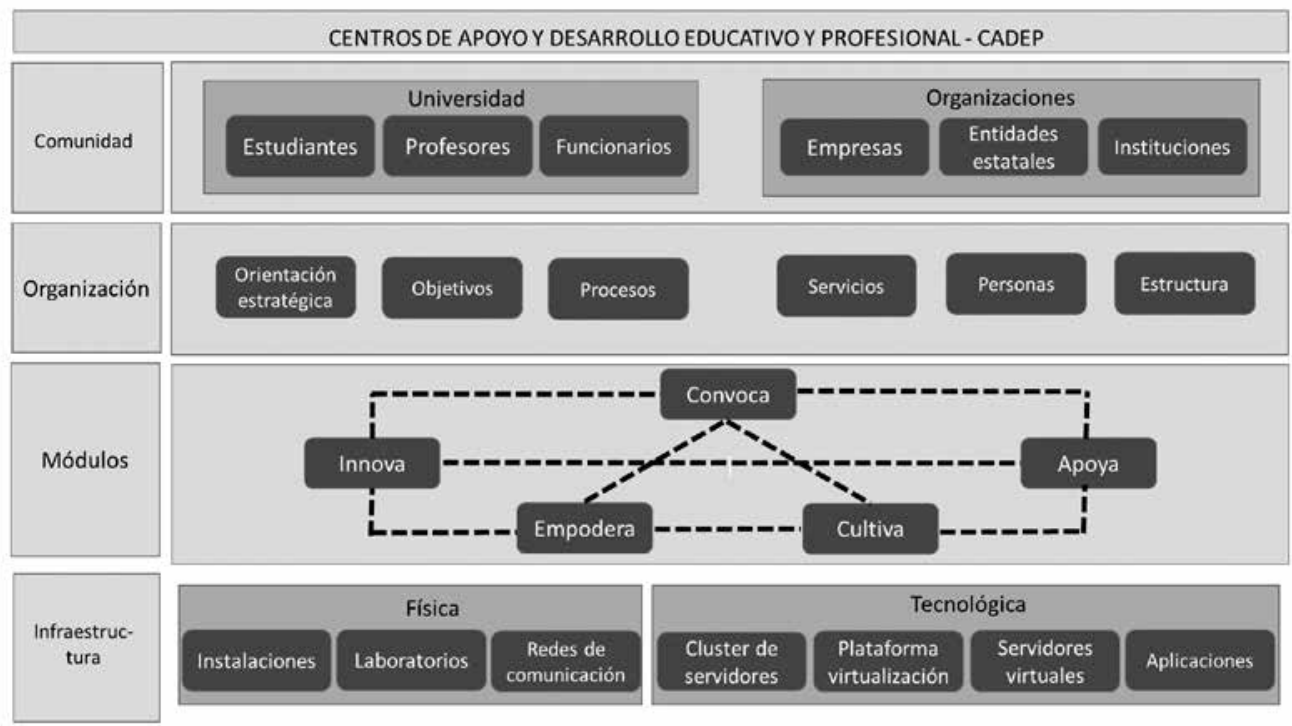

Figura No. 03. Estructura de los centros de apoyo y desarrollo profesional (CADEP) 
El objetivo general de los CADEP se sintetiza en: apoyar, cultivar, adaptar, comunicar, innovar y acoger a la comunidad universitaria en su conjunto, con el fin de favorecer el fomento profesional de todos los miembros de las Instituciones de Educación Superior; la disminución de la deserción estudiantil; la modernización de las estructuras organizativas y para garantizar el respeto por el otro y sus diferencias, a partir de la cooperación intra e interinstitucional en la producción y desarrollo de recursos didácticos y tecnológicos accesibles, y en la generación de estrategias de fomento socioafectivo en atención a las poblaciones en riesgo de exclusión universitaria. Con el fin del cumplimiento de este objetivo general, se desarrollan los objetivos específicos siguientes:

- Articular las dependencias institucionales y la comunidad educativa con un sistema integrado de módulos: Empodera, Innova, Cultiva, Apoya y Convoca, que cumplen funciones de: seguimiento y apoyo a los alumnos en riesgo; formación y apoyo igualmente, tanto al personal académico como al personal técnico y administrativo de la institución; exploración, con sus sistema de laboratorio, de nuevas estrategias para la docencia universitaria; uso innovador de las TIC en las prácticas didácticas y fomento del emprendimiento entre alumnos y profesores, entre otras.

- Desarrollar la autonomía de profesores y personal responsable en la creación y apropiación de contenidos accesibles y personalizables, en ambientes de comunidades de práctica, así como proporcionar las adaptaciones necesarias que supongan una carga indebida para ellos; a partir de la actividad del Módulo Empodera.

- Desarrollar capacidades de innovación en el buen uso, reutilización y creación de aplicaciones y dispositivos orientados a cubrir necesidades educativas específicas de las poblaciones en riesgo de exclusión universitaria, a partir de la actividad del Módulo Innova.

- Desarrollar la competencia didáctica de profesores y estudiantes en el diseño, gestión y apropiación de ambientes de aprendizaje y de propuestas didácticas accesibles que incorporan la afectividad, a partir de los referentes curriculares definidos en el proyecto ALTER-NATIVA, desde la actividad del Módulo Cultiva.

- Desarrollar la competencia emocional e inclusiva con un sistema de detección, seguimiento y apoyo de necesidades emocionales, con actividades de difusión y concientización sobre las diferencias socioculturales y, con un enfoque diferencial para la mejora del rendimiento académico y la reducción del abandono estudiantil, a partir de la actividad del Módulo Apoya.

- Desarrollar las competencias institucionales en gestión de Centros de Apoyo y Desarrollo Educativo Profesional y en cooperación solidaria intra e inter institucional, a partir de la integración de todos los módulos del Centro en la producción y comunicación de información sobre soluciones e investigaciones educativas, a partir de la actividad del Módulo Convoca. 


\section{La innovación en los centros de apoyo y desarrollo educativo profesional}

La principal innovación en los CADEP será un sistema integrado de módulos (Empodera, Innova, Cultiva, Apoya y Convoca) que ofrecerán estrategias y recursos que mejoran el ambiente institucional y ofrecen soluciones innovadoras a profesores, personal técnico, personal administrativo y, estudiantes. En el módulo Empodera se pretende desarrollar la autonomía del profesorado y talentos humanos responsable en la creación y apropiación de contenidos y adaptaciones accesibles y personalizables, en ambientes de comunidades de prácticas. El módulo Innova tiene la finalidad desarrollar las capacidades de innovación en el buen uso, reutilización y creación de aplicaciones y dispositivos orientados a cubrir necesidades educativas específicas de las poblaciones en riesgo de exclusión universitaria. El módulo Cultiva desarrollará la competencia didáctica del profesorado y el estudiantado incorporando los referentes curriculares definidos en el proyecto ALTER-NATIVA del programa europeo ALFA III (Consorcio ALTER-NATIVA, 2013).

El módulo Apoya desarrollará la competencia emocional de la comunidad universitaria con un sistema de detección, seguimiento y apoyo de necesidades emocionales y de reconocimiento y comprensión de las diferencias socioculturales. El módulo Convoca desarrollará las competencias institucionales en gestión de CADEP y en cooperación solidaria intra e inter institucional, a partir de la integración de todos los módulos del centro en la producción y comunicación de información sobre soluciones e investigaciones educativas. Todo ello se llevará a cabo con un enfoque diferencial para la mejora del rendimiento académico y la reducción del abandono estudiantil.

\section{Los cinco módulos suponen diversos tipos de innovación:}

- Innovación en la afectividad académica y social universitaria, mediante soluciones de aprendizaje automático para facilitar el desarrollo de una herramienta de apoyo que detecta las emociones, utilizando entre otros el paradigma de la Internet of Things (IoT), capaces de generar recomendaciones didácticas que respondan a los estados emocionales del estudiante que son detectados y seguidos para mejorar su nivel académico y evitar la deserción estudiantil. También, mediante campañas de sensibilización de la comunidad de alcance de la universidad, incluyendo familia, empresas, y resto del tejido social (Apoya).

- Innovación tecnológica en la docencia universitaria, mediante el uso de sistemas de gestión de conocimiento, así como la creación y reutilización de aplicaciones y dispositivos innovadores, fomentando el emprendimiento universitario, la transferencia de conocimiento y la relación universidad-empresa (innova y empodera).

- Innovación didáctica en ambientes universitarios mediante: el desarrollo de pautas, modelos y prototipos de tipo didáctico y aplicaciones para apoyar procesos de enseñanza y aprendizajes diferenciados según condiciones cognitivas, 
afectivas y culturales de los estudiantes, con miras a disminuir los niveles de repitencia de asignaturas. Acciones como la incorporación de referentes que flexibilizan los currículos universitarios según escenarios y condiciones de aprendizaje de los estudiantes; la actualización de los docentes en cuanto a su autonomía para la creación de contenidos y ambientes de aprendizaje accesibles para atender las necesidades y preferencias de poblaciones con diferencias en el acceso al conocimiento, constituyen innovaciones de tipo didáctico (Cultiva y Empodera).

- Innovación en la gestión académica universitaria mediante un sistema de gestión de conocimiento para la cooperación solidaria, que detecta fomenta y transfiere innovaciones en lo afectivo, en lo tecnológico y en lo didáctico en su sistema de infraestructura física, tecnológica y de comunicación; necesarias para el buen funcionamiento del CADEP y su articulación interna y externa con otras instancias universitarias, otras entidades y otros CADEP (Convoca).

\section{El impacto de los CADEP-Acacia, en la Educación Superior en América y Europa}

El impacto de los CADEP Acacia en la Educación Superior en América y Europa se presenta en los siguientes niveles y grupos de cooperación:

El internacional. Se fortalecen las redes de universidades consolidadas en los programas ALFA, (como la Red ALTER-NATIVA), por la generación de acciones de las 14 universidades miembros de ACACIA, para la sostenibilidad y explotación de los recursos de programas ALFA, en particular los que provienen de ALTER-NATIVA, ESVIAL, y EQUALITY, en el desarrollo de estrategias robustas para la Educación Superior. Las universidades de Europa y ALC, podrán acceder a sistemas de información sobre: relaciones entre disminución de deserción y repitencia; caracterización del efecto de la afectividad social en la formación profesional; tendencias didácticas y tipos de ambientes de aprendizaje en las Universidades; usos innovadores de las tecnologías, y formas de cooperación solidaria intra e interinstitucional. Se consolida así, un observatorio para otro tipo de indicadores de la Educación Superior.

El regional. Se consolida el sistema de CADEP Acacia, como un sistema de creación, formación y reflexión universitaria que fomenta la valoración de cada persona en la universidad y de su necesidad de crecimiento profesional y que reconoce en las didácticas, las tecnologías y la valoración de la afectividad social un potencial para ese crecimiento. Las 11 universidades de ALC tendrán la posibilidad de fortalecer y beneficiarse de los tres CADEP Acacia o crear un nuevo CADEP.

El nacional. Las universidades socias de los países de Chile, Perú, Brasil, Colombia y Nicaragua, dispondrán de equipos de profesionales formados para crear, usar y valorar: recursos; tecnologías; didácticas; ambientes de aprendizaje; y formas de 
cooperación solidaria en comunidades de práctica. Estos equipos y universidades son un recurso para fortalecer estrategias y programas nacionales de mejoramiento de la Educación Superior en cada país y para asesorar a otros grupos en el desarrollo de estrategias similares, así como para participar en mesas de trabajo de los ministerios y secretarias de educación.

El institucional. Se consolida la cooperación solidaria entre las diferentes dependencias administrativas y académicas de las universidades y se ofrece un espacio para el encuentro institucional, la reflexión, la creación y el aprendizaje cooperativo, para aprender haciendo, ofreciendo una alternativa para los cursos de capacitación de docentes o de formación continuada.

El comunitario. Se consolidan comunidades de práctica profesionales con profesores, estudiantes, administrativos, técnicos, investigadores, expertos, familias y miembros de la sociedad, que se asociaron por el proyecto y que van a disponer de recursos y formas de cooperación organizadas, para explorar iniciativas de mejora e innovación en la Educación Superior.

\section{Conclusiones}

La deserción universitaria en Europa, América Latina y el Caribe se ha convertido en un fenómeno que ha puesto a prueba las políticas gubernamentales y de las instituciones de Educación Superior, dado que es un fenómeno complejo que va permeado por múltiples factores que, a su vez, interactúa entre sí para generar riesgos potenciales a la permanencia del estudiantado. Como respuesta, el proyecto ACACIA ofrece un sistema articulado de acciones que cubre no sólo las necesidades de los estudiantes con acciones centradas en sus necesidades y preferencias, sino también con acciones y formación dirigidas tanto al profesorado como al personal técnico y administrativo universitario, sin olvidar a la familia y el entorno social en el que se inscribe la universidad incluyendo al tejido empresarial, con una perspectiva integradora y aplicada de la innovación desarrollada.

En este sentido, ACACIA propone desarrollar centros de apoyo y desarrollo profesional constituidos en un sistema de módulos: Empodera, Innova, Cultura, Apoya y Cultiva, que además integran laboratorios, software, modelos de sistemas, metodologías, cursos, pautas y orientaciones necesarias para el correcto funcionamiento y desenvolvimiento de cada uno de ellos. Todo ello, con la finalidad de:

- Fortalecer el proceso enseñanza y aprendizaje a través de la implementación del modelo pedagógico acorde al proyecto educativo institucional.

- Fortalecer las condiciones que facilitan el proceso de enseñanza y aprendizaje asegurando la calidad y excelencia académica. 
- Fortalecer el sistema de evaluación de los aprendizajes acorde a las normativas académicas existentes.

- Promover el intercambio académico a través de las prácticas y pasantías estudiantiles.

- Promover el desarrollo de resultados de aprendizaje genéricos que garanticen la formación integral estudiantil.

- Contribuir a la calidad docente a través de acciones que garantice las competencias necesarias para la pertinencia académica.

- Promover actividades extracurriculares en la formación integral estudiantil desde sus primeros años de estudios en la universidad.

- Promover a través del CADEP la atención integral al estudiantado para garantizar la retención y promoción.

- Promover un sistema de laboratorios que incorporen acciones pertinentes para la atención de poblaciones en contexto de diversidad.

- Potenciar el proceso educativo con tecnologías accesibles que cubran las necesidades de todos los actores del proceso educativo en contexto de diversidad.

Además, el CADEP, supone un nuevo punto de encuentro y centro de formación y actualización de conocimientos sobre uso y reutilización de tecnologías, creación de contenidos accesibles y generación de las adaptaciones necesarias, didácticas que atienden a las necesidades físicas y sensoriales así como a la afectividad de los alumnos, detección humana y automática de emociones que pueden estar afectando al alumno en su avance académico y personal, así como sistemas de gestión del conocimiento para facilitar la interacción interna de los módulos del CADEP y de su relación con otras instancias universitarias internas y externas.

\section{Lista de referencias}

Aburto, P. (2013). Deserción estudiantil universitaria UNAN-MANAGUA, FAREMCARAZO. Terrón Universitario(5), 84-93.

Bisquerra, R. (2009). Metodología de la Investigación Educativa. Madrid: La Muralla.

Cabrera, L., Bethencourt, J. T., Álvarez Pérez, P., \& González Afonso, M. (2006). El problema del abandono de los estudios universitarios. [The dropout problem in university study]. Revista ELectrónica de Investigación y EValuación Educativa (RELIEVE), 12(2).

Casiano, L. (2004). The realitonship among living situation, health and college academic performance. www.webclearinghouse.net, 7. 


\section{EDUCACIÓN SUPERIOR}

Consorcio ALTER-NATIVA. (2013). Informe final del Proyecto Europeo ALTER-NATIVA - ALFA III. Bogotá: Universidad Distrital Francisco José de Caldas.

Eurostat. (o2 de 03 de 2015). Smarter, greener, more inclusive? - Indicators to support the Europe 2020 strategy. Recuperado el 10 de 06 de 2016, de Eurostat: http:// ec.europa.eu/eurostat/documents/3217494/6655013/KS-EZ-14-oo1-EN-N.pdf/ a5452f6e-819o-4f3o-8996-41b1306f7367

González, L. E. (2005). Repitencia y deserción universitaria en América Latina. Chile: IESALC.

Hernández, R., Fernández, C., \& Baptista, M. (2010). Metodología de la Investigación. México: McGra-Hill.

Lukeš, D. (18 de o8 de 2012). MOOC motivations and magnitudes: Reflections on the MOOC experience vs the MOOC drop out. Recuperado el 15 de 06 de 2016, de Researchity - Exploring Open Research and Open Education: http://researchity. net/2012/o8/18/mooc-motivations-and-magnitudes/

MINEDUC. (2012). Deserción de la eduación superior en Chile. Santiago: Ministerio de Educación. Gobierno de Chile.

Moncada, L. (2014). La integración académica de los estudiantes universitarios como factor determinante del abandono de corto plazo. Un análisis en el Sistema de Educación Superior a Distancia del Ecuador. RIED. Revista Iboeroamericana de Educación a Distancia, 17(2).

Parrino, M. (2014). Factores intervinientes en el fenómeno de la deserción universitaria. Revista Argentina de Educación Superior(8), 36-61.

Programa Alfa. (2012). GUIA - Integrated University Management of Student Dropout ALFA III Projects. Recuperado el 10 de 06 de 2016, de Alfa: http://www.alfa3programme.eu/en/projects/ project/28-GUIA---Integrated-University-Management-of-Student-Dropout

Sierra, H., \& Hernández, O. (2014). Sistema de alertas tempranas como herramienta de innovación tecnológica en la Universidad Santo Tomás para el fortalecimiento de la permanencia estudiantil y graduación oportuna. Conferencia Latinoamericana sobre abandono en la Educación Superior (págs. 496-504). Medellín: CLABES. Obtenido de http://clabes-alfaguia.org/clabes-2014/docs/IVCLABES2014_ LibrodeActas.pdf

Summerskill, J. (1962). Dropouts from College. En N. S. (Ed), The American College (págs. 627-655). New York: Wiley.

Tinto, A. (2009). Deserción estudiantil en la Educación Superior colombiana, metodología de seguimiento, diagnostico y elementos para su prevención. Bogotá: Ministerio de Educación Nacional.

UNESCO. (2014). EFA Global Monitoring Report. 